\title{
Projeto de Trocadores de Calor Casco e Tubo usando o Algoritmo de Polinização de Flores e Modelos Caóticos
}

\author{
Design of Shell-and-Tube Heat Exchanger using \\ Flower Pollination Algorithm and Chaotic Pattern
}

Gustavo B. Libotte ${ }^{1}$, Fran S. Lobato², Gustavo M. Platt ${ }^{3}$, Francisco D. Moura Neto ${ }^{4}$

\section{RESUMO}

Nas últimas décadas, inúmeras estratégias para a atualização dinâmica dos parâmetros nos algoritmos heurísticos têm sido propostas. Estas têm como objetivo aumentar a capacidade de exploração do algoritmo e, consequentemente, evitar a convergência para ótimos locais e minimizar a influência dos parâmetros de entrada que, tradicionalmente são considerados constantes durante o processo evolutivo. Neste contexto, este trabalho tem como objetivo empregar modelos caóticos de busca para a atualização de um dos parâmetros do Algoritmo de Polinização de Flores. A metodologia proposta foi empregada para o projeto de trocadores de calor do tipo casco e tubo em que se deseja determinar variáveis geométricas para fins da minimização do custo total. Os resultados obtidos com a aplicação da metodologia proposta em dois estudos de caso permitem concluir que a inserção de um modelo caótico de busca aumentou a capacidade de exploração do algoritmo canônico, bem como permitiu a redução do número de avaliações da função-objetivo.

Palavras-chave: Trocador de Calor Casco e Tubo, Algoritmo de Polinização de Flores, Modelo Caótico, Projeto de Sistemas de Engenharia.

\section{ABSTRACT}

In the last decades, numerous strategies to update dynamically the heuristic algorithms parameters have been proposed. These approaches aim to increase the exploration capacity of the algorithm and, consequently, avoid convergence to local optima and to minimize the influence of input parameters that are traditionally considered constant during the evolutionary process. In this context, this work aims to use chaotic search models in order to update one of parameters of Flower Pollination Algorithm. The proposed methodology was used in design of shell-and-tube heat exchanger in which it is desired to determine geometric variables to minimize the total cost. The results obtained with the application of such methodology in two case studies allow the conclusion that the insertion of a chaotic search model increased the exploration capacity of the canonical algorithm, as well as the reduction of number of objective function evaluations.

Keywords: Shell-and-Tube Heat Exchanger, Flower Pollination Algorithm, Chaotic Pattern, Engineering System Design.
1,4 Instituto Politécnico,

Universidade do Estado do Rio de Janeiro, Nova Friburgo, RJ, 28630050, Brasil

E-mail:

${ }^{1}$ gustavolibotte@iprj.uerj.br

${ }^{4}$ fmoura@iprj.uerj.br

${ }^{2}$ Faculdade de Engenharia

Química, Universidade Federal

de Uberlândia, Av. João Naves de

Ávila 2121 - Campus Santa

Mônica, Bloco 1K, CEP 38408-

144, Uberlândia, MG, Brasil.

E-mail: fslobato@ufu.br

${ }^{1}$ Grupo de Engenharia e Otimização de Processos

Industriais, Escola de Química e Alimentos, Universidade Federal do Rio Grande, Santo Antônio da Patrulha, RG, 95500000, Brasil.

E-mail: gmplatt@furg.br 


\section{INTRODUÇAOO}

O projeto de trocadores de calor configura uma área de grande interesse industrial e acadêmico. Isto se deve, entre outros fatores, ao grande número de aplicações que podem ser desenvolvidas com este tradicional equipamento, dentre as quais pode-se citar os processos de geração de energia, de integração energética e indústrias petroquímicas. Entre os vários tipos de trocadores de calor que existem, os denominados de casco e tubo são os mais utilizados, sendo que, nas indústrias, mais de $65 \%$ dos trocadores são deste tipo (Shah e Dusan, 2003). A escolha por tal tipo de configuração se deve ao fato destes maximizarem a relação área/volume, sendo que para os gases pode-se alcançar a relação $700 \mathrm{~m}^{2} / \mathrm{m}^{3}$ e para os líquidos pode-se alcançar $300 \mathrm{~m}^{2} / \mathrm{m}^{3}$, bem como aos custos relacionados com a sua construção (Mohanty, 2016).

Em se tratando do projeto, o problema a ser analisado envolve um grande número de variáveis geométricas e operacionais, bem como equações constitutivas e correlações empregadas para a modelagem fenomenológica do mesmo. Neste caso, três são os objetivos que, costumeiramente, são empregados na etapa de projeto, a saber, a minimização do custo total, maximização da razão área/volume da troca de calor e a maximização da efetividade (Mohanty, 2016). Neste contexto, Selbas et al. (2006) determinaram a configuração ótima de trabalho de um trocador de calor do tipo casco e tubo usando os tradicionais Algoritmos Genéticos. Neste estudo, os autores consideravam três variáveis de projeto (todas relacionadas com a geometria do trocador) para fins da minimização do custo total por ano, que incluía o investimento de capital e despesas operacionais. Nos anos seguintes, vários outros estudos considerando diferentes estratégias também foram empregadas para a resolução deste mesmo estudo de caso. Neste cenário, pode-se citar o uso do Particle Swarm Optimization (Patel e Rao, 2010), do Artificial Bee Colony (Sahin et al., 2011), do Cuckoo Search (Asadi et al., 2014), do Imperialist Competitive Algorithm (Hadidi et al., 2013), do Biogeography-Based Algorithm (Hadidi, A. Nazari, 2013) e do Firefly Algorithm (Mohanty, 2016). Em todas estas aplicações, observa-se que o valor da função-objetivo, isto é, da minimização do custo total, sempre foi reduzido, sendo que, a depender do tipo de fluido quente e frio considerado, o valor da redução pode chegar a $70 \%$.

Dentre as últimas estratégias de otimização propostas, o Algoritmo de Polinização de Flores (APF) (do inglês, Flower Pollination Algorithm), configura-se como um dos mais empregados e estudados. Esta estratégia, proposto por Yang (2012) para resolver 
problemas de otimização, é inspirado no processo de polinização de flores por meio de insetos via definição de movimento segundo a distribuição de Lévy (Pavlyukevich, 2007).

Desde a primeira publicação do APF, vários trabalhos têm sido apresentados. Dentre estes, pode-se destacar a otimização de problemas multi-objetivos considerando a associação entre o APF e o método da soma ponderada (Yang et al., 2013, 2014); a otimização de estruturas (Bekdas et al, 2015); a localização ótima de antenas (Dahi et al., 2016); a otimização de funções matemáticas a partir da hibridização do APF com o Algoritmo de Seleção Clonal (Nabil, 2016); a otimização de funções matemáticas considerando novos operadores de mutação (Salgotra e Singh, 2017); determinação de parâmetros em modelos de bioimpedância (Yousri, 2018).

No APF, pode-se destacar como um dos principais parâmetros, a probabilidade de troca. Este valor é responsável por direcionar o processo de otimização como sendo um de busca local (refinamento da solução ótima corrente) ou busca global (capacidade de exploração do espaço de projeto). Conforme descrito por Yang (2012), a escolha deste parâmetro é um dos pontos cruciais do método, visto que ao se definir por um ou outro valor, pode-se fazer com que a população caminhe em direção a um ótimo local ou até mesmo que tenha convergência prematura, isto é, perca a sua capacidade de exploração do espaço de projeto. No algoritmo proposto Yang (2012), este parâmetro é considerado constante durante todo o processo evolutivo, sendo que o valor de 0,8 é considerado como uma boa estimativa para o mesmo, de acordo com o próprio autor.

Nas últimas décadas, modelos caóticos de busca têm sido empregados como alternativa para a atualização dinâmica dos parâmetros nos algoritmos heurísticos. Isto se deve ao fato de que a associação entre modelos caóticos de busca e os métodos de otimização aumenta a capacidade de exploração do espaço de projeto, o que na prática minimiza a chance de convergência prematura (Li e Jiang, 1998). Como exemplos, podese citar a associação entre diferentes modelos caóticos com o algoritmo de Evolução Diferencial e com o algoritmo de Programação Quadrática Sucessiva (Coelho e Mariani, 2006). Já Tavazoei e Haeri (2007) realizaram um estudo sobre a eficácia de modelos caóticos de busca associados a métodos clássicos de otimização. Nesta mesma linha, também pode-se citar Yang et al. (2007), que propõem combinações entre o Método de Monte Carlo, o Algoritmo Clássico BFGS (Broyden-Fletcher-Goldfarb-Shanno) (Vanderplaats, 1999) e alguns modelos caóticos para formar algoritmos híbridos.

Diante do que foi apresentado, a presente contribuição tem por objetivo propor uma nova metodologia para o projeto de um trocador de calor do tipo casco e tubo para fins da 
minimização do custo total. A metodologia proposta consiste no uso de modelos caóticos de busca para atualizar a probabilidade de troca no APF. Este trabalho está estruturado como segue: na Seção 2, serão apresentados aspectos gerais sobre o problema de interesse; na Seção 3 é apresentada a descrição do APF e, por sua vez, a metodologia proposta neste trabalho é descrita na Seção 4. Os resultados são apresentados na Seção 5. Finalmente, as conclusões são apresentadas na última seção.

\section{MODELAGEM MATEMÁTICA DO PROBLEMA DE INTERESSE}

A Figura 1 apresenta um diagrama esquemático de um trocador de calor do tipo casco e tubo onde deseja-se promover a troca de calor entre um fluido quente e um fluido frio (Incropera e DeWitt, 2002).

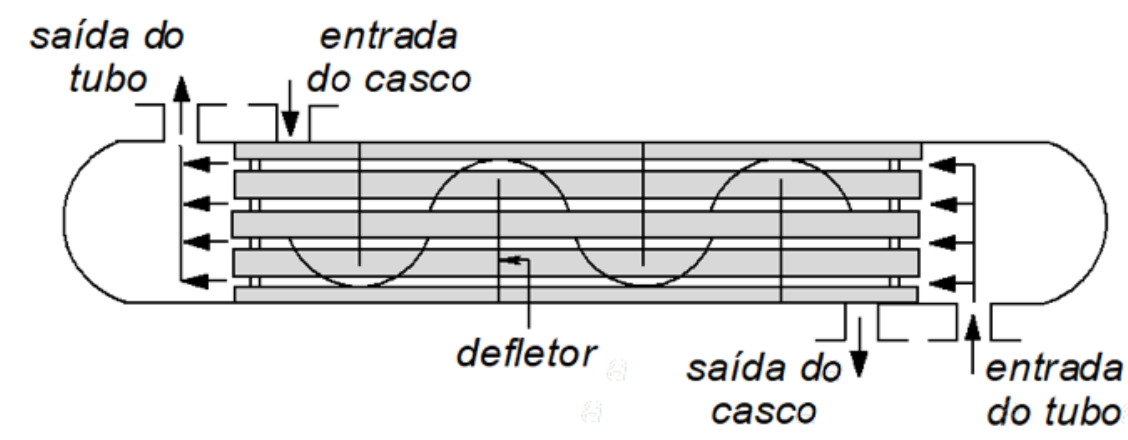

Figura 1: Representação esquemática do trocador de calor do tipo Casco e tubo.

Em linhas gerais, este equipamento é constituído de um feixe de tubos montados em um casco cilíndrico, em que uma corrente quente, que entra no casco (ou no tubo), troca calor com uma corrente fria que entra nos tubos (ou no casco). Neste caso, a maximização do calor trocado depende da definição das condições de operação, tais como as temperaturas de entrada, a pressão de trabalho do trocador, das características do fluido, da incrustação de material ao longo da operação e da configuração geométrica do trocador (Mohanty, 2016).

Matematicamente, o modelo que representa o mecanismo de transferência de calor neste tipo de equipamento é composto por uma série de equações que relacionam as variáveis dependentes e independentes. A seguir é descrito o modelo de interesse neste trabalho estudado por vários autores (Selbas et al., 2006; Caputo et al., 2008; Asadi et al., 2014; Mohanty, 2016).

Dependendo do regime de fluxo, o coeficiente de transferência de calor referente ao tubo $\left(h_{t}\right)$ é calculado como (Caputo et al., 2008): 


$$
h_{t}=\left\{\begin{array}{l}
\frac{k_{t}}{d_{i}}\left(3,657+\frac{0,0677\left(\operatorname{Re}_{t} \operatorname{Pr}_{t}\left(d_{i} / L\right)\right)^{1,33}}{1+0,1 \operatorname{Pr}_{t}\left(\operatorname{Re}_{t}\left(d_{i} / L\right)\right)^{0,3}}\right) ; \quad \operatorname{Re}_{t}<2300 \\
d_{i}\left(1+12,7\left(f_{t} / 8\right)^{0,5}\left(\operatorname{Pr}_{t}^{2 / 3}-1\right)\right. \\
0,027 \frac{k_{t}}{d_{i}} \operatorname{Re}_{t} 0,8 \operatorname{Pr}_{t} 1 / 3\left(\frac{\mu_{t}}{\mu_{w t}}\right)^{0,14} ; \operatorname{Re}_{t}>10000
\end{array}\right.
$$

em que $k_{t}$ representa a condutividade térmica do tubo, $d_{i}$ é diâmetro interno do tubo, $L$ é o comprimento dos tubos, $\mu_{t}$ e $\mu_{w t}$ representam a viscosidade dinâmica no interior do tubo e na parede, respectivamente, $\operatorname{Re}_{t}$ e $\operatorname{Pr}_{t}$ representam os número de Reynolds e de Prandtl em relação ao tubo, respectivamente, e $f_{t}$ é o fator de atrito de Darcy do tubo, definido como sendo (Hewitt, 1989):

$$
f_{t}=\left(1,82 \log \left(\operatorname{Re}_{t}\right)-1,64\right)^{-2}
$$

$\operatorname{Re}_{t}$ e $\operatorname{Pr}_{t}$ são calculados como:

$$
\begin{aligned}
\operatorname{Re}_{t} & =\frac{\rho_{t} v_{t} d_{i}}{\mu_{t}} \\
\operatorname{Pr}_{t} & =\frac{\mu_{t} C p_{t}}{k_{t}}
\end{aligned}
$$

em que $\rho_{t}$ é densidade, $C p_{t}$ é a capacidade calorífica, ambos referentes ao tubo. Já a velocidade do fluxo no tubo $\left(v_{t}\right)$ é dado por (Sinnott et al., 1996):

$$
v_{t}=\frac{m_{t}}{(\pi / 4) d_{t}^{2} \rho_{t}}\left(\frac{n}{N_{t}}\right)
$$

em que $n$ é o número de passes e $N_{t}$ é o número de tubos, definidos por (Sinnott et al., 1996):

$$
N_{t}=K_{1}\left(\frac{D_{s}}{d_{0}}\right)^{n_{1}}
$$

tal que $K_{1}$ e $n_{1}$ são coeficientes referentes ao tipo de configuração considerada (triangular ou quadrangular), como apresentado na Fig. 2 e definidos na Tab. 1 (Rohsenow e Hartnett, 1973; Sinnott et al., 1996; Shah e Bell, 2000). 


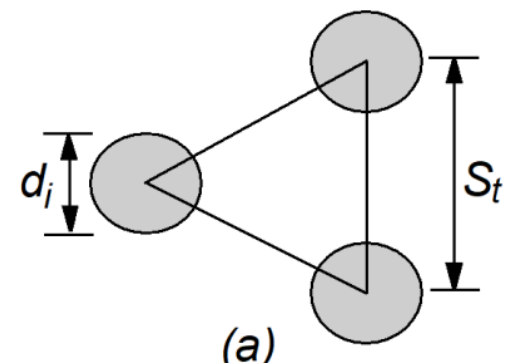

(a)

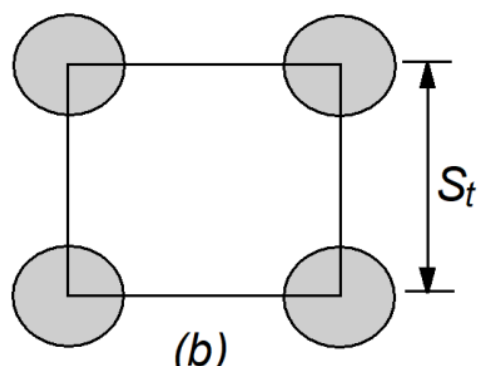

(b)

Figura 2: Tipos de Configurações: (a) Triângulo e (b) Quadrado. Em que $d_{o}$ é diâmetro externo do tubo e $S_{t}$ é a distância entre os tubos.

Tabela 1. Valores dos coeficientes $K_{1}$ e $n_{1}$ para diferentes tipos de arranjos.

Número de Configuração do Tipo Triangular Configuração do Tipo Quadrangular

\begin{tabular}{ccccc} 
Passes & \multicolumn{2}{c}{$S_{i=1,25} d_{0}$} & $K_{1}$ & $S_{1}=1,25 d_{0}$ \\
\hline 1 & 0,319 & 2,142 & 0,215 & 2,207 \\
2 & 0,249 & 2,207 & 0,156 & 2,291 \\
4 & 0,175 & 2,285 & 0,158 & 2,263 \\
6 & 0,0743 & 2,499 & 0,0402 & 2,617 \\
8 & 0,0365 & 2,675 & 0,0331 & 2,643 \\
\hline
\end{tabular}

O coeficiente de transferência de calor referente ao casco $\left(h_{s}\right)$ é dado como (Kern, 1950):

$$
h_{s}=0,36 \frac{k_{t}}{d_{e}} \operatorname{Re}_{s}^{0,55} \operatorname{Pr}_{s}^{1 / 3}\left(\frac{\mu_{s}}{\mu_{w t s}}\right)^{0,14}
$$

em que $d_{e}$ é o diâmetro hidráulico do casco (Sinnott et al., 1996):

$$
d_{e}=\left\{\begin{array}{l}
\frac{4\left(S_{t}^{2}-\left(\pi d_{O}^{2} / 4\right)\right)}{\pi d_{O}} ; \text { configuração quadrada } \\
\frac{4\left(0,43 S_{t}^{2}-\left(0,5 \pi d_{O}^{2} / 4\right)\right)}{0,5 \pi d_{O}} ; \text { configuração triangular }
\end{array}\right.
$$

Por sua vez, o número de Prandtl para o casco é dado por:

$$
\operatorname{Pr}_{S}=\frac{\mu_{S} C p_{S}}{k_{S}}
$$

e considerando $m_{s}$ como o fluxo mássico no casco, a velocidade do fluxo $v_{s}$ no casco é definida como (Selbas et al., 2006):

$$
v_{S}=\frac{m_{S}}{\rho_{S} A_{S}}
$$

Já a área da seção transversal normal ao sentido de fluxo $\left(A_{s}\right)$ é dado por Selbas et al. (2006): 


$$
A_{S}=D_{S} B\left(1-\frac{d_{O}}{S_{t}}\right)
$$

em que $D_{s}$ é o diâmetro interno do casco e $B$ é o espaçamento dos defletores.

O número de Reynolds para o casco é obtido como:

tal que $m_{s}$ é o fluxo mássico no casco.

$$
R e_{S}=\frac{m_{S} d_{e}}{A_{S} \mu_{S}}
$$

Dessa forma, coeficiente global de transferência de calor $(U)$ é dado como (Incropera e DeWitt, 2002):

$$
U=\frac{1}{\frac{1}{h_{s}}+R_{f S}+\left(\frac{d_{0}}{d_{i}}\right)\left(R_{f t}+\frac{1}{h_{t}}\right)}
$$

onde $h_{s}$ é o coeficiente de transferência de calor referente ao casco, $R_{f s}$ e $R_{f t}$ representam as resistências de incrustação referentes ao casco e ao tubo, respectivamente, e $d_{i}=0,8 d_{0}$.

A temperatura média logarítmica $\left(\Delta T_{L M}\right)$ é determinada como:

$$
\Delta T_{L M}=\frac{\left(T_{h i}-T_{C O}\right)-\left(T_{h o}-T_{C i}\right)}{\ln \left(\left(T_{h i}-T_{C O}\right) /\left(T_{h o}-T_{C i}\right)\right)}
$$

em que $T_{h i}$ e $T_{\text {ho }}$ representam as temperaturas de entrada e saída do fluido quente, respectivamente, $T_{c i}$ e $T_{c o}$ representam as temperaturas de entrada e saída do fluido frio, respectivamente.

Dependendo da configuração do fluxo e do tipo de casco, é necessário aplicar um fator de correção $(F)$, introduzido para calcular a área de superfície do trocador de calor $(A)$. Este fator é dado como (Incropera e DeWitt, 2002):

$$
F=\sqrt{\frac{R^{2}+1}{R-1}} \frac{\ln ((1-P) /(1-P R))}{\ln \left(\left(2-P R+1-\sqrt{R^{2}+1}\right) /\left(2-P R+1+\sqrt{R^{2}+1}\right)\right)}
$$

onde $R$ é um coeficiente de correção, definido como:

$$
R=\frac{T_{h i}-T_{h o}}{T_{c o}-T_{c i}}
$$

e $P$ representa a eficiência do processo, descrito como:

$$
P=\frac{T_{C O}-T_{C i}}{T_{h i}-T_{C i}}
$$

Neste caso, a área de troca térmica é dada pela seguinte equação: 


$$
A=\frac{Q}{U F \Delta T_{L M}}
$$

Já o comprimento do tubo pode ser calculado de acordo com a seguinte relação:

$$
L=\frac{A}{\pi d_{O} N_{t}}
$$

Para o projeto do trocador de calor, faz-se necessário definir uma função-objetivo. Para esta finalidade, considera-se a minimização do custo total por ano ( $\left.\mathrm{C}_{\text {tot }}\right)$ como funçãoobjetivo. Esta inclui o investimento de capital $\left(C_{i}\right)$, o custo de energia $\left(C_{e}\right)$ e o custo operacional anual $\left(C_{o p}\right)$ (Caputo et al., 2008). Matematicamente, o custo total é definido como:

$$
C_{\text {tot }}=C_{i}+C_{o p}
$$

O investimento de capital é calculado em função da área de transferência de calor (Tall et al., 2003):

$$
C_{i}=a_{1}+a_{2} A^{a 3}
$$

onde os parâmetros $a_{i}$ são definidos como sendo iguais a $8000 ; 259,2$ e 0,91, respectivamente (considera-se o aço inoxidável como material para o casco e para os tubos). Considerando que o dinheiro ganho em um ano pode ser reinvestido, essa recapitalização pode ser calculada através de uma variação da fórmula de juros compostos. Portanto, o custo operacional pode ser obtido pela seguinte equação:

$$
\begin{gathered}
C_{o p}=\sum_{j=1}^{n_{y}} \frac{P_{1} C_{e} H}{(1+i)^{j}} \\
P_{1}=\frac{1}{\eta}\left(\frac{m_{t}}{\rho_{t}} \Delta P_{t}+\frac{m_{S}}{\rho_{s}} \Delta P_{s}\right)
\end{gathered}
$$

em que $C_{e}$ é igual a 0,00012; $H$ (tempo total de operação por ano) é igual a 7000; $i$ (taxa de desconto anual) é igual a 0,1 e $\eta$ (eficiência global de bombeamento) é igual a 0,8. O fluxo de caixa líquido estimado em cada ano do projeto (representado pelo produto $\mathrm{P}_{1} \mathrm{C}_{\mathrm{e}} \mathrm{H}$ ) é trazido ao seu "valor presente" no início do projeto, descontando-o alguma taxa de juros, que é escolhida para refletir o poder aquisitivo do dinheiro. Seria aproximadamente equivalente à taxa de juros atual que o dinheiro poderia render se fosse investido (Sinnott et al., 1996).

Nesse contexto, $\Delta P_{t}$ e $\Delta P_{s}$ representam a queda de pressão no tubo e no casco, respectivamente. A primeira é calculada como a soma da queda de pressão distribuída ao 
longo do comprimento do tubo e das perdas de pressão concentrada nos cotovelos, bem como nos bicos de entrada e saída, conforme a seguinte equação (Kern, 1950):

$$
\Delta P_{t}=\frac{\rho_{t} v_{t}^{2}}{2}\left(\frac{L}{d_{i}} f_{t}+4\right) n
$$

Já a queda de pressão no casco é dada por:

$$
\Delta P_{S}=f_{S}\left(\frac{\rho_{S} v_{S}^{2}}{2}\right)\left(\frac{L}{B}\right)\left(\frac{D_{S}}{d_{e}}\right)
$$

onde $f_{s}$ é o fator de atrito, definido como sendo igual a (Peters e Timmerhaus, 1991):

$$
f_{S}=1,44 \operatorname{Re}_{S}^{-0,15}
$$

Apresentado o problema de projeto, na próxima seção será descrito o APF, empregado como metodologia base para a resolução deste estudo de caso.

\section{ALGORITMO DE POLINIZACCAOO DE FLORES}

\subsection{A Inspiração Biológica}

O processo de polinização é um dos mais importantes na natureza, sendo este de fundamental importância para a evolução da fauna e da flora. Em linhas gerais, o processo de polinização nas flores se dá pela transferência de pólen via contribuição de diferentes espécies, como por exemplo, insetos, pássaros, morcegos, entre outros (Glover, 2007). Este tipo de polinização, denominada de biótica, compreende cerca de $90 \%$ das plantas. Já os outros 10\% restantes pertencem a classe abiótica e se processa, basicamente, pela ação do vento e da sua difusão na água (Glover, 2007; Yang, 2012).

Uma característica interessante dos agentes polinizadores e que a relaciona com um processo evolutivo é que estes tendem a visitar certas espécies de flores, ignorando outras. Neste caso, a transferência de pólen de flores para uma mesma espécie é maximizada, o que implica na reprodução de determinadas espécies de flores (Yang, 2012). Essa transferência pode ser realizada via autopolinização ou por polinização cruzada. A primeira acontece a partir da transferência de pólen de uma flor de uma planta diferente, enquanto que na autopolinização, a fertilização é realizada a partir de uma mesma flor (Glover, 2007). Assim, a associação entre estes dois mecanismos de polinização representa, na natureza, a capacidade dos agentes polinizadores percorrerem longas distâncias, o que do ponto de vista da otimização, é uma característica bem interessante (caracterizada como polinização global) (Yang, 2012). Para a realização da polinização com o auxílio de insetos, faz-se 
necessário definir um tipo de movimento que, neste caso, pode ser modelado via distribuição de Lévy (Pavlyukevich, 2007).

\subsection{Passos do APF}

A partir das características citadas, Yang (2012) propôs o APF, cuja concepção conceitual pode ser resumida nas seguintes etapas:

1. A polinização biótica e cruzada é considerada como um processo global de polinização, em que os polinizadores se comportam como modelo de distribuição de Lévy;

2. A polinização abiótica e a autopolinização são consideradas como uma etapa que corresponde a polinização local;

3. A polinização local e a polinização global são controladas por uma probabilidade de troca $p$ pertencente ao intervalo $[0,1]$;

4. Devido à proximidade física e outros fatores, como o vento, a polinização local pode ter uma fração significativa $p$ nas atividades globais de polinização;

5. Para fins de simplificação, cada planta tem apenas uma única flor e cada flor pode liberar apenas uma unidade de pólen.

No APF, podem ser destacados dois passos principais, a saber, as polinizações global e local. Na global, os pólens das flores são carregados por polinizadores, podendo estes viajar por longas distâncias. Isso garante a polinização e a reprodução das flores mais aptas (candidatos à solução do problema de otimização). Matematicamente, este procedimento pode ser descrito como segue:

$$
x_{i}^{t+1}=x_{i}^{t}+L(\lambda)\left(x_{i}^{t}-g_{\text {best }}\right)
$$

em que $x_{i}^{t}$ é o $i$-ésimo pólen (vetor de solução $x_{i}$ na $t$-ésima iteração ou geração), $g_{\text {best }}$ é a melhor solução encontrada até a t-ésima geração, $L(\lambda)$ representa o tamanho do passo durante a polinização (os insetos podem se mover por longas distâncias com diferentes tamanhos de passos). Esse parâmetro é determinado a partir da avaliação da distribuição Lévy, isto é:

$$
L \square \frac{\lambda \Gamma(\lambda) \sin (\pi \lambda / 2)}{\pi} \frac{1}{s^{1+\lambda}}, \quad(s \square 0)
$$

em que $\Gamma(\lambda)$ é a função gama padrão e $s$ é um número aleatório com distribuição normal.

A polinização local pode ser representada pela seguinte relação: 


$$
x_{i}^{t+1}=x_{i}^{t}+\varepsilon\left(x_{j}^{t}-x_{k}^{t}\right)
$$

em que $x_{j}^{t}$ e $x_{k}{ }^{t}$ representam pólens de diferentes flores de uma mesma espécie de planta, respectivamente. Do ponto de vista matemático, se $x_{j}^{t}$ e $x_{k}^{t}$ vêm da mesma espécie ou se são selecionados da mesma população, tem-se uma busca em que o caminho é puramente aleatório e ponderado por um número aleatório $\varepsilon$ com distribuição uniforme entre $[0,1]$. Assim, para ocorrer a alternância entre as buscas global e local, faz-se necessário a utilização de um parâmetro auxiliar, denominado de probabilidade de troca $p$ (também conhecida como probabilidade de proximidade). Assim, caso seja gerado um valor aleatório menor que este valor, procede-se à busca global, e caso contrário, tem-se a busca local (Yang, 2012).

A Tabela 2 apresenta o algoritmo canônico do APF (Yang, 2012):

\section{Algoritmo da Polinização de Flores}

\section{Início}

Definir a função-objetivo $J(x), x=\left(x_{1}, x_{2}, \ldots, x_{n}\right)^{\top}$

Definir os parâmetros do APF (tamanho da população NP e probabilidade de troca $p$ ) Inicializar a população aleatoriamente e determinar a melhor solução corrente ( $\left.g_{\text {best }}\right)$

Enquanto o número de gerações máximo não é alcançado, faça

Para $i=1: n$

Se rand $<p$,

Gere um novo candidato via polinização global, através da Eq. (27)

\section{Senão}

Gere um novo candidato via polinização local, utilizando a Eq. (29)

\section{Fim Se}

Avalie novas soluções de acordo com a função-objetivo e armazene a melhor

\section{Fim Para}

\section{Fim Enquanto}

Imprima os resultados

Tabela 2: Pseudocódigo para o AFP.

Neste algoritmo, o usuário deve fornecer a função-objetivo, o número de variáveis de projeto $(n)$, o número de flores $(N P)$, a probabilidade de troca $(p)$, o valor do parâmetro $\lambda$ (geralmente adotado como sendo igual a 1,5), e o número máximo de gerações $\left(N_{\text {gen }}\right)$. 


\section{METODOLOGIA}

Como descrito anteriormente, em qualquer método de otimização clássico ou heurístico, a definição dos parâmetros de entrada, para a grande maioria das aplicações, é um fator que influência o processo de convergência. Neste contexto, para a atualização da probabilidade de troca $(p)$ no APF, serão considerados modelos caóticos de busca para essa finalidade. Assim, considere os seguintes modelos (DEVANEY, 1987; OTT, 2002):

$$
\begin{gathered}
x_{i+1}=\mu x_{i}\left(1-x_{i}\right) \quad(3,57 \leq \mu \leq 4) \\
x_{i+1}=\frac{\mu}{4} \sin \left(\pi x_{i}\right) \quad(0 \leq \mu \leq 4)
\end{gathered}
$$

em que $x$ é o parâmetro que se deseja atualizar e $\mu$ é uma constante que define 0 comportamento caótico para cada modelo. Na prática, conhecendo-se o valor inicial para a probabilidade de troca, a mesma pode ser atualizada durante o processo evolutivo de acordo com as Eqs. (30) e (31). O APF associado a um modelo caótico de busca será denominado de APF+MCB.

Para a presenta aplicação, definiu-se como variáveis de projeto o diâmetro externo do tubo $\left(d_{o}\right)$, o diâmetro interno do casco $\left(D_{s}\right)$ e o espaçamento entre os defletores $(B)$. Já a função-objetivo é definida na Eq. (20) e representa a minimização do custo total.

Em linhas gerais, o problema do projeto do trocador de calor pode ser resumido nas seguintes etapas:

1. Definir os parâmetros do APF e os parâmetros considerados para a simulação do trocador de calor;

2. Gerar, de forma aleatória e segundo os limites estabelecidos no APF, a população inicial. Esta é avaliada de acordo com o modelo do trocador de calor, sendo que a melhor solução (vetor de variáveis de projeto e função-objetivo) é tomada como sendo o ótimo. Cabe ressaltar que, para a avaliação da função-objetivo, o seguinte procedimento é adotado:

- Entrar com o vetor de variáveis de projeto definidas pelo APF+MCB;

- Definir as características do fluido quente (casco) e frio (tubos), bem como condições operacionais;

- Calcular o diâmetro interno do tubo $\left(d_{l=0,8}=0 d_{0}\right)$, a constante de $\operatorname{Sinnot}\left(S_{t}=1,25 d_{o}\right)$, a temperatura média logarítmica, o coeficiente de correção $R$, a eficiência do processo $P$, o fator de correção $F$ e o calor transferido $Q$;

- A partir destas informações, inicializa-se um laço iterativo cuja finalidade é o cálculo do coeficiente global de transferência de calor (U). Para essa finalidade 
inicializa-se este procedimento fazendo com que $U$ seja igual a $300 \mathrm{~W} /\left(\mathrm{m}^{2} \mathrm{~K}\right)$ (estimativa inicial - $U_{o}$ ). Em seguida determina-se a área de troca térmica $A, 0$ número de tubos $\left(N_{t}\right)$, a velocidade do fluxo no tubo $\left(V_{t}\right)$, o número de Reynolds no tubo $\left(\operatorname{Re}_{t}\right)$, o número de Prandtl no tubo $\left(\operatorname{Pr}_{t}\right)$, o fator de atrito de Darcy $\left(f_{t}\right)$, o comprimento dos tubos $(L)$, o coeficiente de transferência de calor convectivo do tubo $\left(h_{t}\right)$, o diâmetro hidráulico $\left(D_{e}\right)$, a área da seção cruzada $\left(A_{s}\right)$; o número de Reynolds no casco $\left(\operatorname{Re}_{s}\right)$, o número de Prandtl no casco $\left(\operatorname{Pr}_{s}\right)$, o coeficiente de transferência de calor convectivo no casco $\left(h_{s}\right)$ e o novo valor para $U$. Caso a diferença relativa entre $U$ e $U_{0}$ seja maior do que 0,3 (conforme sugerido por Sinnott et al. (1996)), o procedimento continua e, caso contrário, o valor do coeficiente global de transferência de calor é encontrado;

- Com o valor de $U$ determina-se a queda de pressão no tubo $\left(\Delta P_{t}\right)$, o fator de atrito $\left(f_{s}\right)$, a velocidade do fluxo no casco $\left(v_{s}\right)$, a de pressão no casco $\left(\Delta P_{s}\right)$, e, consequentemente, o valor do custo total anual.

3. Atualizar o valor da probabilidade de troca e proceder à busca local ou global;

4. Atualizar a população atual;

5. Repetir o procedimento até que a população se torne homogênea, isto é, a diferença relativa acumulada entre os indivíduos da população seja menor do que $10^{-6}$. Caso contrário, o APF é executado até que o número máximo de gerações seja alcançado.

Para avaliar os resultados obtidos pela metodologia proposta, considera-se quatro diferentes combinações de parâmetros para o $\operatorname{APF}$, a saber: $\operatorname{APF}(1)(p=0,8)$; $\operatorname{APF}(2)(p$ como sendo um número aleatório definido no intervalo $[0,1])$; $\operatorname{APF}(3)$ ( $p$ sendo atualizado via Eq. (30)); e APF(4) ( $p$ sendo atualizado via Eq. (31)). Em todos os casos, foi escolhido $N P=25$.

\section{RESULTADOS E DISCUSSĀO}

Nessa seção serão apresentados dois estudos de caso para validar a metodologia proposta. No primeiro estudo de caso será avaliado um trocador de calor com metanol e água salobra, considerando a representação triangular da Fig. 2(a), com um passe no casco e dois passes no tubo. Já no segundo é considerado um trocador de calor com querosene e óleo bruto, com um passe no casco e quatro passes no tubo, com configuração quadrada, como mostrado na Fig. 2(b). 
Em ambos os casos, o custo total será avaliado no decorrer de $n_{y}=10$ anos e taxa de desconto percentual igual a $i=0,1$. Inicialmente, serão apresentados os melhores resultados $\left(J_{b}\right)$, os piores $\left(J_{w}\right)$ e os valores médios $\left(J_{a}\right)$ da objetivo-objetivo, além do número médio de avaliações da função-objetivo (NAFO) e a redução percentual $(\Gamma)$, tomando como referência o maior NAFO, cujos valores foram obtidos através do APF, utilizando as quatro combinações de parâmetros estipuladas anteriormente, para 100 execuções distintas sob as mesmas condições.

Em seguida, os resultados obtidos através do Particle Swarm Optimization (Patel e Rao, 2010), Cuckoo Search (Asadi et al., 2014) e Firefly Algorithm (Mohanty, 2016), relacionados ao valor ótimo das variáveis de projeto $\left(X^{*}\right)$, valor da função-objetivo correspondente $\left(J\left(X^{*}\right)\right)$, número médio de avaliações da função-objetivo (NAFO) e a redução percentual $(\Gamma)$, tomando como referência o maior NAFO dentre as técnicas avaliadas, serão comparados.

O custo total será avaliado considerando duas contribuições, a saber, o custo do investimento e o custo de operação. Para essa análise, também serão avaliados os resultados obtidos pelas três metaheurísticas citadas anteriormente.

Em todos os casos, as variáveis de projeto foram avaliadas considerando os seguintes intervalos de busca: diâmetro interno do casco $\left(D_{s}\right)$ variando de $0,1 \mathrm{~m}$ a $1,5 \mathrm{~m}$; diâmetro externo dos tubos $\left(d_{0}\right)$ variando de $0,015 \mathrm{~m}$ a $0,051 \mathrm{~m}$; espaçamento dos defletores $(B)$ variando de $0,05 \mathrm{~m}$ a 0,5 $\mathrm{m}$. A Tabela 1 apresenta as propriedades físicas de cada um dos elementos contidos no trocador de calor em ambos os estudos de caso.

Tabela 1. Propriedades físicas dos elementos para ambos os estudos de caso (Asadi et al., 2014).

\begin{tabular}{ccccc}
\hline & \multicolumn{2}{c}{ Estudo de caso 1 } & \multicolumn{2}{c}{ Estudo de caso 2 } \\
\hline & Casco: metanol & Tubo: água salobra & Casco: querosene & Tubo: óleo bruto \\
\hline$m(\mathrm{~kg} / \mathrm{s})$ & 27,8 & 68,9 & 5,52 & 18,8 \\
$T_{i}\left({ }^{\circ} \mathrm{C}\right)$ & 95,0 & 25,0 & 199,0 & 37,8 \\
$T_{o}\left({ }^{\circ} \mathrm{C}\right)$ & 40,0 & 40,0 & 93,3 & 76,7 \\
$\rho\left(\mathrm{kg} / \mathrm{m}^{3}\right)$ & 750,0 & 995,0 & 850,0 & 995,0 \\
$C_{p}(\mathrm{~kJ} / \mathrm{kg} \mathrm{K})$ & 2,84 & 4,2 & 2,47 & 2,05 \\
$\mu(\mathrm{Pa} \mathrm{s})$ & 0,00034 & 0,0008 & 0,0004 & 0,00358 \\
$\mu_{\mathrm{w}}(\mathrm{Pa} \mathrm{s})$ & 0,00038 & 0,00052 & 0,00036 & 0,00213 \\
$k(\mathrm{~W} / \mathrm{m} \mathrm{K})$ & 0,19 & 0,59 & 0,13 & 0,13
\end{tabular}


DOI 10.18605/2175-7275/cereus.v11n1p117-139
LIBOTTE, G. B.; LOBATO, F. S.; PLATT, G. M.; MOURA NETO, F. D.

Projeto de Trocadores de Calor Casco-Tubo usando o

Algoritmo de Polinização de Flores e Modelos Caóticos
$R_{\mathrm{f}}\left(\mathrm{m}^{2} \mathrm{~K} / \mathrm{W}\right)$
0,00033
0,0002
0,00061
0,00061

\subsection{Metanol-Água}

A Tabela (2) apresenta os resultados obtidos considerando a aplicação das quatro abordagens propostas. Em relação ao NAFO, a estratégia $\operatorname{APF}(2)$ foi a que demandou 0 maior valor, já as estratégias APF(3) e APF(4) foram as com menor valor de avaliações da função-objetivo, seguida pela APF(1). Neste caso, considerando-se o parâmetro $p$ como sendo atualizado aleatoriamente, não foi possível, para o problema em análise, reduzir o esforço computacional. Por outro lado, assumindo que o mesmo é constante, o número de avaliações reduz-se, mas não tão quanto o parâmetro é atualizado considerando-se um modelo caótico de busca. É importante ressaltar que o caráter aleatório do parâmetro também pode fazer com que o algoritmo realize uma busca refinada no início das gerações, mas não foi capaz de fazer com que a população se torne homogênea, visto que o número total de gerações foi alcançado. Assim, a atualização do parâmetro através de mapas caóticos faz com que a convergência ocorresse com menos gerações, isto é; a população se tornou homogênea. Em termos do valor da função-objetivo, ambas as estratégias obtiveram resultados semelhantes. Todavia, os melhores resultados médios foram obtidos considerando as estratégias $\operatorname{APF}(3)$ e $\operatorname{APF}(4)$, o que demonstra a capacidade de exploração do algoritmo com a atualização dinâmica do parâmetro $p$ considerando modelos caóticos.

Tabela 2. Resultados obtidos para o estudo de caso 1 considerando as quatro abordagens propostas.

\begin{tabular}{ccccc}
\hline & APF(1) & APF(2) & APF(3) & APF(4) \\
\hline$J_{b}$ & 45633,24 & 45633,24 & 45633,24 & 45633,17 \\
$J_{w}$ & 45715,79 & 45677,34 & 45652,55 & 45671,39 \\
$J_{a}$ & 45641,69 & 45643,38 & 45635,01 & 45635,89 \\
NAFO & 5125 & 6025 & 4550 & 4750 \\
$\Gamma(\%)$ & 85,06 & 100 & 75,52 & 78,84 \\
\hline
\end{tabular}

A seguir, serão avaliados os valores ótimos obtidos por cada uma das metaheurísticas da literatura, em relação àqueles calculados pela técnica abordada nesse trabalho. Nos trabalhos em que as versões canônicas do Particle Swarm Optimization (Patel e Rao, 2010) e o Cuckoo Search (Asadi et al., 2014) foram utilizados na otimização do 
DOI 10.18605/2175-7275/cereus.v11n1p117-139
LIBOTTE, G. B.; LOBATO, F. S.; PLATT, G. M.; MOURA NETO, F. D.

Projeto de Trocadores de Calor Casco-Tubo usando o

Algoritmo de Polinização de Flores e Modelos Caóticos

problema, não foram informados, de maneira explícita, o NAFO. No primeiro deles, os autores reportam que o número de partículas escolhido foi 50 e o algoritmo obteve o resultado ótimo após 100 gerações sendo executado. Sabe-se que, a cada geração, o Particle Swarm Optimization realiza tantas avaliações da função-objetivo quanto forem o número de partículas da população. Portanto, pode-se inferir que o algoritmo realizou, pelo menos, 5050 avaliações da função-objetivo, uma vez que a população inicial também é avaliada.

No caso do Cuckoo Search, os autores também não reportam o NAFO necessário até a convergência, tampouco o número de indivíduos da população. Analogamente ao caso anterior, é sabido que o algoritmo Cuckoo Search realiza duas avaliações (em condições distintas), de cada um dos indivíduos da população corrente, a cada geração. Considerando que o algoritmo obteve as soluções ótimas após 100 gerações, como ilustrado no trabalho em questão, e que a população inicial de pássaros também deve ser avaliada antes do início do processo iterativo, foram necessárias, pelo menos, NP + $2 \times \mathrm{NP}$ x 100 avaliações da função-objetivo. A Tabela (3) apresenta os resultados relacionados aos ótimos obtidos por cada um dos métodos citados, além do Firefly Algorithm e dos resultados correspondentes ao melhor valor mostrado na Tabela (2).

Tabela 3. Valores ótimos obtidos para o estudo de caso 1 considerando diferentes estratégias evolutivas.

\begin{tabular}{ccccc}
\hline & PSO & CS & FA & APF \\
\hline \multicolumn{5}{c}{$\left(d_{o} D_{s} B_{0}\right)$} \\
\hline$X^{*}$ & $(0,0150,810,424)$ & $(0,01510,8260,414)$ & $(0,015750,8580,402)$ & $(0,0150,8960,5)$ \\
$J\left(X^{*}\right)$ & 53231,1 & 47625,1 & 45782,0 & 45633,24 \\
NAFO & 5050 & $N P+2 \times N P \times 100$ & 4000 & 4550 \\
$\Gamma(\%)$ & 100 & $(201 / 5050) \times 100 \times N P$ & 79,21 & 90,1 \\
\hline
\end{tabular}

Analisando os valores obtidos, em comparação àqueles da literatura, nota-se uma tendência acerca das variáveis de projeto: nas variáveis de projeto ótimas, obtidas através de todos os métodos analisados, o diâmetro externo dos tubos sempre foi escolhido como um valor próximo ao seu limite inferior no intervalo de busca. As demais variáveis de projeto também se mantiveram próximas, com o espaçamento dos defletores mais próximo ao limite superior de busca e o diâmetro interno do casco com resultados variando entre, aproximadamente, 0,8 e 0,9. Esses fatos confirmam a correção do modelo implementado 
e o seu alto grau de não-linearidade, vista a diferença no valor da função-objetivo, em relação às soluções relativamente próximas.

Ainda em relação à Tabela (3), o Particle Swarm Optimization obteve os valores ótimos das variáveis de projeto que configuram o maior custo total dentre as metodologias comparadas, mesmo com o maior valor absoluto do NAFO. Obviamente, o método Cuckoo Search pode apresentar custo computacional aproximadamente mais elevado do que o Particle Swarm Optimization para os casos em que NP $\geq 25$. No caso da metodologia abordada nesse trabalho, o NAFO foi 10,89\% mais alto do que o Firefly Algorithm, o algoritmo que apresentou os melhores resultados quanto ao custo computacional, dentre as metodologias levadas em conta nessa análise. Em contrapartida, pode-se notar um ganho no valor da função-objetivo obtida pelo APF em relação ao FA.

\subsection{Querosene-Óleo}

Analogamente ao que foi apresentado anteriormente, no segundo estudo de caso, estudado por Kern (1950), deseja-se obter os valores ótimos das variáveis de projeto que minimizem o custo total do trocador de calor, calculado pela Eq. (20). A Tabela (4) apresenta os resultados obtidos para cada uma das abordagens relacionadas às combinações de parâmetros do APF.

Tabela 4. Resultados obtidos para o estudo de caso 2 considerando as quatro abordagens propostas.

\begin{tabular}{ccccc}
\hline & APF(1) & APF(2) & APF(3) & APF(4) \\
\hline$J_{b}$ & 19444,83 & 19444,83 & 19444,83 & 19444,83 \\
$J_{w}$ & 19448,15 & 19448,16 & 19447,58 & 19447,71 \\
$J_{a}$ & 19446.68 & 19446,71 & 19445,23 & 19445,05 \\
NAFO & 4025 & 4725 & 3550 & 3425 \\
$\Gamma(\%)$ & 85,19 & 100 & 75,13 & 72,49 \\
\hline
\end{tabular}

Nesta tabela observa-se que todas as quatro estratégias alcançaram o valor mínimo do custo total de operação em, pelo menos, uma das execuções. Além da redução do NAFO, pode-se notar que o valor médio da função-objetivo, dentre 100 execuções típicas, também foi mais próximo do valor mínimo quando o algoritmo foi executado utilizando a atualização dinâmica do parâmetro $p$ através de mapas caóticos. Ainda, essas estratégias propiciaram valores com desvio-padrão menos acentuados em relação às execuções de 
DOI 10.18605/2175-7275/cereus.v11n1p117-139
LIBOTTE, G. B.; LOBATO, F. S.; PLATT, G. M.; MOURA NETO, F. D.

Projeto de Trocadores de Calor Casco-Tubo usando o

Algoritmo de Polinização de Flores e Modelos Caóticos

APF(1) e APF(2), visto que a amplitude entre os valores extremos da função-objetivo (Jb e $J_{w}$ ) obtidos durante os testes foram menores.

$\mathrm{Na}$ Tabela (5) observa-se valores similares para as variáveis de projeto $d_{0}$ e $B_{0}$ e próximos aos seus respectivos limites, mas diferentes valores para a variável de projeto $D_{s}$, o que implica de uma mudança neste valor não resultou em uma mudança significativa para a função-objetivo, demonstrando a complexidade do problema em questão. Nesse estudo de caso, o APF com a estratégia de atualização dinâmica do parâmetro $p$ através do mapa caótico da Eq. (31) apresentou convergência para o ponto ótimo com um menor número de avaliações da função-objetivo em relação ao FA, mesmo que a solução obtida represente um custo total moderadamente acima daquele obtido pelo FA. No caso das demais metaheurísticas presentes na comparação, os valores foram os mesmos apresentados na análise do primeiro caso, sempre com custo computacional acima das demais abordagens.

Tabela 5. Valores ótimos obtidos para o estudo de caso 2 considerando diferentes estratégias evolutivas.

\begin{tabular}{ccccc}
\hline & PSO & CS & FA & APF \\
\hline \multicolumn{5}{c}{$\left(d_{o} D_{s} B_{o}\right)$} \\
\hline$X^{*}$ & $(0,0150,63$ & $(0,0150,590,1112)$ & $(0,015750,7276$ & $0,1054)$ \\
& $0,1118)$ & $10,01500,7318$ & $0,1104)$ \\
$J\left(X^{*}\right)$ & 19922,6 & NP $+2 \times \mathrm{NP} \times 100$ & 19433 & 19444,83 \\
NAFO & 5050 & $(201 / 5050) \times 100 \times$ & 4000 & 3425 \\
$\Gamma(\%)$ & 100 & NP & 79,21 & 67,82 \\
\hline
\end{tabular}

\subsection{Comparativo Econômico}

Do ponto de vista econômico, o projeto do trocador de calor pode ser visto sob duas perspectivas distintas: o custo total de manutenção do trocador de calor compreende os custos de investimento, representados por $C_{i}$ e calculados pela Eq. (21), e os custos relacionados à operação, denotado por $C_{o p}$ e obtidos através da Eq. (22). O primeiro está diretamente à área de superfície do trocador de calor, que pode variar o seu custo, por exemplo, de acordo com o número de tubos e a configuração (triangular ou quadrada) escolhida. No caso dos custos relacionados a operação, a eficiência energética e as quedas máximas de pressão permitidas são algumas das variáveis que afetam os gastos durante a operação. A Fig. (3) apresenta cada um desses custos discriminados, calculados por cada um dos métodos utilizados para análise. 
A análise dos custos referente ao primeiro estudo de caso (ver a Fig. (3a)) explicita a diferença entre os dois melhores resultados obtidos pelas metaheurísticas analisadas. Mesmo que os custos totais sejam semelhantes, nota-se considerável dissimilaridade entre os custos de investimento e de operação obtidos por cada uma dessas estratégias de otimização. Os valores ótimos obtidos pelo APF propiciaram queda de pressão menos acentuada, tanto do lado do casco quanto do tubo, permitindo que o custo operacional fosse mais baixo. Em contrapartida, o equilíbrio dos custos se deu por conta do valor superior em relação ao custo de investimento, que está diretamente relacionado aos parâmetros referentes à área de troca térmica, calculados pela Eq. (18).

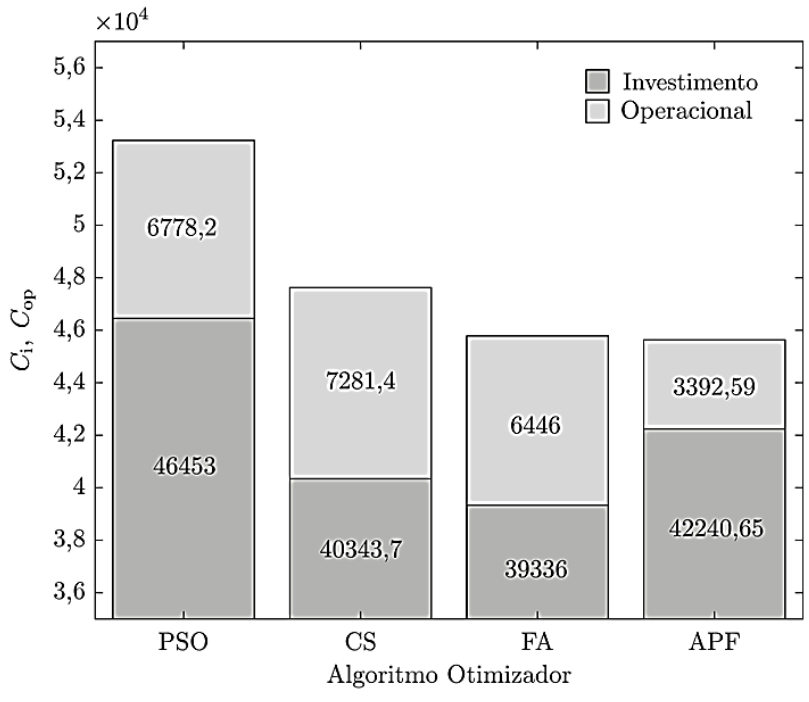

(a) Metanol-Água Salobra.

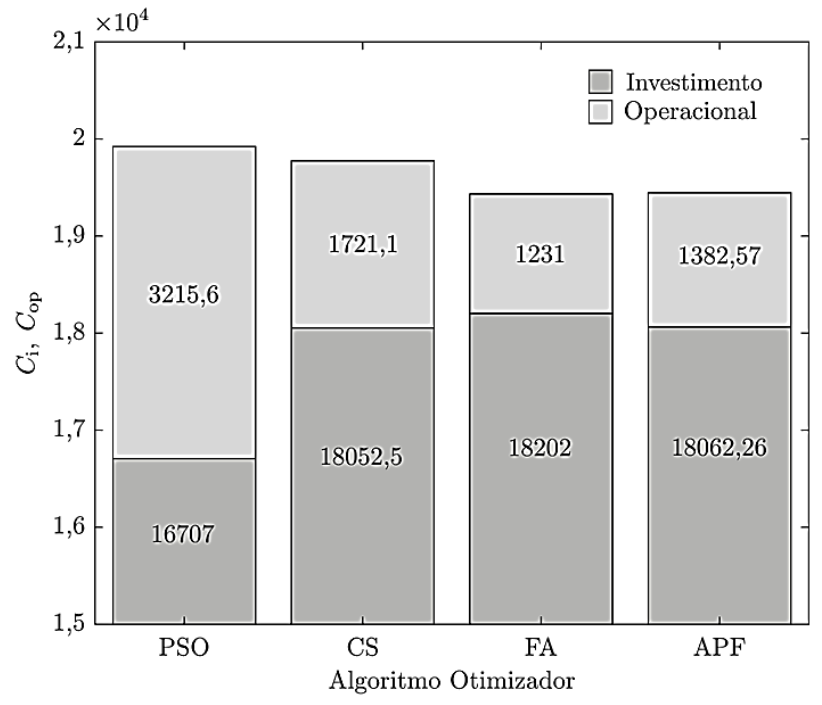

(b) Querosene-Óleo Bruto.

Figura 3: Avaliação do custo de investimento $\left(C_{\mathrm{i}}\right)$ em relação ao custo operacional $\left(C_{\mathrm{op}}\right)$ obtido por cada uma das metaheurísticas, para ambos os estudos de caso.

Já para o segundo estudo de caso (ver a Fig. (3b)) observa-se que o FA e o APF convergiram para soluções próximas, se comparados os valores ótimos mostrados na Tabela 5. Além disso, os valores dos custos de investimento e operacional foram semelhantes, mas com margem de mais de $10 \%$ em relação ao custo computacional estimado através do número de avaliações da função-objetivo. Possivelmente, parte dessa ligeira diferença entre os resultados analisados pode se dar pelo fato de que os valores ótimos listados nos trabalhos considerados nessa comparação foram truncados. Eventualmente, os algoritmos em questão poderiam convergir a mesma solução, com uma quantidade limitada de casas decimais, se a execução de ambos fosse definida sob os mesmos critérios de parada. 


\section{CONCLUSÓES}

Este trabalho apresentou duas alternativas para a atualização dinâmica do parâmetro seletor de tipo de polinização do Algoritmo de Polinização de Flores, a saber, usando modelos caóticos de busca. As técnicas foram testadas para a obtenção das soluções ótimas de um modelo de trocador de calor de casco e tubo. Como observado, os modelos caóticos apresentaram melhoria na convergência do algoritmo, fazendo com a solução ótima fosse obtida através de quantidade reduzida de gerações, em relação a maioria das soluções apresentadas na literatura corrente.

Além disso, também foi observado que a atualização dinâmica do parâmetro em questão fez com que a taxa de obtenção das soluções ótimas no problema estudado aumentasse, uma vez que a manutenção de tal parâmetro como valor fixo não é propícia para a diversificação dos indivíduos da população. Em geral, uma grande vantagem dessa abordagem é que ela não acrescenta custo computacional significativo ao processo iterativo, fazendo com que o método obtenha resultados de maneira mais eficiente através de um procedimento simples.

Obviamente, a proposta desse estudo não tem como objetivo apontar uma abordagem que prevaleça sobre as demais. A complexidade de se afirmar que uma estratégia é mais apropriada do que outras é muito grande, uma vez que se deve constatar que a metodologia comparada obtém resultados melhores, de um ponto de vista generalizado em relação a todas as demais técnicas do mesmo tipo. Nesse sentido, os resultados obtidos mostram que a atualização dinâmica do parâmetro $p$ considerando modelos caóticos oferece uma alternativa à utilização do valor constante, como proposto no algoritmo original, obtendo-se resultados coerentes e, possivelmente, significando melhoria em alguns aspectos, como redução do número de avaliação da função-objetivo até a convergência.

Principalmente no primeiro estudo de caso, quando houve grande diferença em relação aos custos de investimento e de operação entre os dois melhores resultados comparados, dependendo da necessidade de operação e da frequência de atividade do trocador de calor, pode ser mais interessante colocar em prática uma estrutura que propicie um custo operacional mais baixo. A longo prazo, essa diferença pode significar um ganho em relação ao custo total.

Como sequência deste trabalho, pretende-se expandir o modelo de trocador de calor para o contexto multi-objetivo considerando os conceitos de robustez e de confiabilidade. 


\section{AGRADECIMENTOS}

Os autores agradecem ao Conselho Nacional de Desenvolvimento Científico e Tecnológico (CNPq), Coordenação de Aperfeiçoamento de Pessoal de Nível Superior (CAPES) e Fundação Carlos Chagas Filho de Amparo à Pesquisa do Estado do Rio de Janeiro (FAPERJ) pelo suporte financeiro necessário para a realização deste trabalho. F. S. Lobato também agradece à Fundação de Amparo à Pesquisa do Estado de Minas Gerais (FAPEMIG).

\section{REFERÉNCIAS}

ASADI, M., SONG, Y., SUNDEN, B., XIE, G. Economic Optimization Design of Shelland-Tube Heat Exchangers by a Cuckoo-Search-Algorithm, Appl. Therm. Eng., 73, 1032-1040, 2014.

BEKDAS, G., NIGDELI, S.M., YANG, X.-S. Sizing Optimization of Truss Structures using Flower Pollination Algorithm, Applied Soft Computing, 37, 322-331, 2015.

CAPUTO, A.C., PELAGAGGE, P.M., SALINI, P. Heat Exchanger Design Based on Economic Optimization, Applied Thermal Engineering, 28, 1151-1159, 2008.

COELHO, L.S., MARIANI, V.C. Combining of Chaotic Differential Evolution and Quadratic Programming for Economic Dispatch Optimization with Valve-Point Effect. IEEE Transactions on Power Systems, 21, 2, 989-996, 2006.

DAHI, Z.A.E.M., MEZIOUD, C., DRAA, A. On the Efficiency of the Binary Flower Pollination Algorithm: Application on the Antenna Positioning Problem, Applied Soft Computing, 47, 395-414, 2016.

HADIDI, A., HADIDI, M., NAZARI, A. A New Design Approach for Shell-and-Tube Heat Exchangers using Imperialist Competitive Algorithm (ICA) from Economic Point of View, Energy Convers. Manage, 67, 66-74, 2013.

HADIDI, A., NAZARI, A. Design and Economic Optimization of Shell-and-Tube Heat Exchangers using Biogeography-Based (BBO) Algorithm, Appl. Therm. Eng., 51,12631272, 2013.

HEWITT, G.F. Heat Exchanger Design Handbook, Begell House, New York, NY, USA, 1989.

INCROPERA, F.P., DEWITT, D.P. Fundamentals of Heat and Mass Transfer, John Wiley and Sons, New York, 2002.

KERN, D.Q. Process Heat Transfer, McGraw-Hill, New York, NY, USA, 1950. 
LI, B., JIANG, W. Optimization of Complex Functions by Chaos Search. International Journal of Cybernetics and Systems, 29, 4, 409-419, 1998.

MOHANTY, D.K. Application of Firefly Algorithm for Design Optimization of a Shell and Tube Heat Exchanger from Economic Point of View. International Journal of Thermal Sciences, 102, 228-238, 2016.

NABIL, E. A Modified Flower Pollination Algorithm for Global Optimization, Expert Systems with Applications, 57, 192-203, 2016.

PATEL, V.K., RAO, R.V. Design Optimization of Shell-and-Tube Heat Exchanger using Particle Swarm Optimization Technique, Appl. Therm. Eng. 30, 1417-1425, 2010.

PAVLYUKEVICH, I. Lévy Flights, Non-Local Search and Simulated Annealing. Journal of Computational Physics, 226, 1830-1844, 2007.

PETERS, M.S., TIMMERHAUS, K.D. Plant Design and Economics for Chemical Engineers, McGraw-Hill, New York, NY, USA, 1991.

ROHSENOW, W.M., HARTNETT, J.P. Handbook of Heat Transfer, McGraw-Hill, New York, 1973.

SAHIN, A.S., KILIÇ, B., KILIÇ, U. Design and Economic Optimization of Shell and Tube Heat Exchangers using Artificial Bee Colony (ABC) Algorithm, Energy Convers. Manage, 52, 3356-3362, 2011.

SALGOTRA R., SINGH, U. Application of Mutation Operators to Flower Pollination Algorithm, Expert Systems with Applications, 79, 112-129, 2017.

SELBAS, R., KIZILKAN, Q., REPPICH, M. A New Design Approach for Shell-and-Tube Heat Exchanger using Genetic Algorithms from Economic Point of View. Chem. Eng. Process, 45, 2567-25772006.

SHAH, R.K., DUSAN, P.S. Fundamentals of Heat Exchanger Design, John Wiley and Sons, New York, 2003.

SINNOTT, R.K., COULSON, J.M., RICHARDSON, J.F. Chemical Engineering Design, Edition 6, Butterworth-Heinemann, Boston, USA, 1996.

SHAH, R.K., BELL, K.J. CRC Handbook of Thermal Engineering, CRC Press, Florida, 2000.

TALL, M., BULATOV, I., KLEMES, J., STEHLIK, P. Cost Estimation and Energy Price Forecast for Economic Evaluation of Retrofit Projects, Appl. Therm. Eng., 23, 18191835, 2003.

TAVAZOEI, M.S., HAERI, M. Comparison of Different One-Dimensional Maps as Chaotic Search Pattern in Chaos Optimization Algorithms. Applied Mathematics and Computation, 187, 1076-1085, 2007. 
VANDERPLAATS, G.N. Numerical Optimization Techniques for Engineering Design. Colorado Springs, CO, Third Edition, 1999.

YANG, X.-S. Flower Pollination Algorithm for Global Optimization. In Unconventional Computation and Natural Computation, edited by J. Durand-Lose and N. Jonoska, Vol. 7445 of Lecture Notes in Computer Science, 240-249. Berlin: Springer, 2012.

YANG, D., LI, G., CHENG, G. On the Efficiency of Chaos Optimization Algorithms for Global Optimization. Chaos, Solutions and Fractals, 34, 1366-1375, 2007.

YANG, X-S., KARAMANOGLU, M., HE, X. Multi-objective Flower Algorithm for Optimization, Procedia Computer Science 18, 861-868, 2013.

YANG, X-S, KARAMANOGLU, M., HE, X. Flower Pollination Algorithm: A Novel Approach for Multiobjective Optimization, Engineering Optimization, 46, 9, 1222-1237, 2014.

YOUSRI, D.A., ABDELATY, A.M., SAID, L.A. Chaotic Flower Pollination and Grey Wolf Algorithms for parameter extraction of bio-impedance models, Applied Soft Computing Journal, 2018 (in press). 